\title{
Evaluation de la neutralité organoleptique des huiles raffinées à l'aide d'un nez électronique
}

\section{Assessing organoleptic level of refined oils using an electronic nose}

Oléagineux, Corps Gras, Lipides. Volume 9, Numéro 4, 264-9, Juillet - Août 2002, Fondamental

Auteur(s) : Pierre-Yves VIGNERON, Joost VANHEMELRIJCK, Bernard STOCLIN, Joël CAIGNIEZ, LESIEUR R \& D Centre, 101, route de Bourbourg, 59412 Coudekerque-Branche, France.

Author(s) : Pierre-Yves VIGNERON, Joost VANHEMELRIJCK, Bernard STOCLIN, Joël CAIGNIEZ

Résumé : Aucune technique analytique ne permet d'évaluer en contrôle industriel si une huile raffinée est de goût neutre c'est à dire dépourvue d'un goût spécifique. La détermination des composés volatils totaux par chromatographie en phase gazeuse de l'espace de tête ne parvient pas à répondre à ce problème puisque cette technique bien que rapide donne des résultats qui ne sont pas corrélés avec la note globale donnée par un panel de dégustation. Les nez électroniques équipés de semi-conducteurs donnent des résultats encourageants dans le cas des huiles de tournesol mais les capteurs actuellement disponibles sont insensibles aux flaveurs de certaines huiles comme les huiles de colza. Quoique de tels équipements ne puissent remplacer à ce jour l'évaluation sensorielle, ils peuvent être un contrôle complémentaire intéressant pour alléger le Contrôle Qualité quotidien d'une production d'huile raffinée.

Summary : No standard analytical techniques are efficient enough to evaluate the bland taste of refined oils in quality control. The determination of total volatile compounds by gas liquid chromatography fails to reach this objective because there is no correlation between the amount and the average score of the taste panel. Electronic noses equipped with metal oxide sensors give promising results mainly for sunflower oils. Although such equipments cannot replace flavor panel evaluation at present time, they could be a very useful tool for the quality control of a daily production of refined oils

Mots-clés : évaluation organoleptique, neutralité de goût des huiles raffinées, détermination des composés volatils, nez électronique, capteurs à oxyde métallique.

Keywords : organoleptic evaluation, blandness of refined oils, volatiles determination, electronic nose, metal oxyde sensor. 


\section{ARTICLE}

Les producteurs d'huiles végétales raffinées et de nombreux chercheurs ont essayé de prédire les caractéristiques organoleptiques des huiles à l'aide de tests chimiques. II en existe un grand nombre parmi lesquels certains sont couramment utilisés comme l'indice de peroxyde, l'indice de paraanisidine, les diènes conjuguées... [1].

Cependant il apparaît qu'aucun n'est capable de caractériser la qualité organoleptique des huiles et de les classer en catégorie « acceptable » « limite » « non acceptable « [2].

Cela n'est pas étonnant puisque les composés " primaires " d'oxydation tels que les peroxydes sont dépourvus de goût et d'odeur et que certains composés " secondaires " d'oxydation dosés par l'indice de para-anisidine ou l'acide thiobarbiturique peuvent avoir une flaveur différente de la flaveur globale. Ainsi une huile de colza peut avoir un net fruité de poisson malgré un indice de peroxyde inférieur à 1 meq $\mathrm{O}_{2} / \mathrm{kg}$ tandis qu'une huile de tournesol peut ne présenter aucun défaut organoleptique même avec un indice de peroxyde de 5 meq $\mathrm{O}_{2} / \mathrm{kg}$.

L'évaluation sensorielle de l'huile paraît être la seule méthode capable de définir si le goût est acceptable ou non puisque le palais humain est sensible à quelques mug/kg de composés aromatiques. Cependant cette technique est assez contraignante; elle nécessite d'abord de disposer d'un panel d'évaluation sensorielle régulièrement entraîné. De plus on constate que la sensibilité des panélistes est différente selon le type de flaveur et que de nombreux facteurs peuvent intervenir sur la représentativité des résultats (nombre et ordre des échantillons, moment de la journée...).

Dans le cas des huiles à goût telles que les huiles vierges et les huiles aromatisées, l'évaluation organoleptique est plus complexe du fait que le profil aromatique est composé d'un grand nombre de molécules, certaines ayant un seuil de détection organoleptique très bas tandis que la flaveur résultante provient du mélange de différentes molécules diluées dans l'air.

C'est pourquoi par souci de simplification, nous avons limité notre étude aux seules huiles raffinées. Dans ce cas, le consommateur attend une huile dépourvue d'un goût spécifique, dite de goût neutre. Le contrôle qualité industriel inclut dans les spécifications le contrôle quotidien du goût de l'huile afin de s'assurer que la désodorisation a été correctement réalisée et que le goût de l'huile après stockage est resté neutre. L'organisation de tests d'analyse sensorielle quotidiens n'est pas facile et est contraignante. Aussi nous avons recherché si d'autres méthodes plus commodes étaient capables de déterminer rapidement si l'huile était dépourvue d'un goût spécifique.

Deux techniques ont été examinées, l'une consistant à déterminer la teneur en composés volatils par chromatographie en phase gazeuse, l'autre employant un nez électronique.

Des échantillons de différentes qualités d'huiles (arachide colza tournesol) provenaient d'une installation industrielle de raffinage. Une partie était redésodorisée dans un désodoriseur de laboratoire conçu en interne. Les conditions de désodorisation étaient effectuées sur 3 litres d'huile à $220^{\circ} \mathrm{C} 1 \mathrm{mbar}$ pendant $3 \mathrm{~h} 30$ [3]. 
Les huiles raffinées en usine et les huiles correspondantes redésodorisées étaient placées en flacon de verre fermé de $250 \mathrm{ml}$ avec un espace vide résiduel de $15 \mathrm{ml}$ avant de subir un vieillissement accéléré.

Le test de vieillissement a été mené sur les échantillons conservés à température ambiante exposés à la lumière naturelle dans une pièce orientée à l'ouest durant 10 semaines, un témoin étant conservé au congélateur.

Chaque semaine, un flacon était retiré et placé au congélateur pour les analyses et l'évaluation sensorielle. Ces conditions de vieillissement proviennent de plusieurs années d'expérience interne montrant une assez bonne corrélation avec la stabilité organoleptique de I'huile en vieillissement naturel à l'obscurité avant l'expiration de sa date limite d'utilisation.

\section{Évaluation sensorielle}

L'évaluation sensorielle des huiles était effectuée par un panel de contrôle qualité industriel pratiquant ce type de mesure quotidiennement. L'objectif est de rechercher si l'huile est neutre de goût ou s'il reste des flaveurs agréables ou désagréables perceptibles et à partir de ce critère, de classer l'huile en « acceptable », « limite », « non acceptable ».

Le mode opératoire consiste à chauffer l'huile à $38^{\circ} \mathrm{C} \pm 2{ }^{\circ} \mathrm{C}$ pendant 30 min avant l'évaluation par 7 panélistes expérimentés disposant chacun d'une vingtaine de millilitres. La dégustation se fait dans une salle équipée de boxes isolés, éclairés par une lumière spécifique ne permettant pas de différencier les échantillons par leur couleur. Chaque boxe dispose d'un écran sur lequel apparaît une page rassemblant 7 descripteurs : fruité verdure ou haricot plastique rance poisson brûlé autres.

Les panélistes évaluent sur une ligne sans échelle l'intensité de chaque descripteur puis à la page suivante, ils attribuent de la même façon une note d'appréciation globale de la qualité de l'huile.

L'ensemble de ces informations transmis à un ordinateur est traité par un logiciel FIZZ (Bio-Systèmes Courtenau France) et exprimé en chiffres (échelle 0-10). La note globale est prépondérante pour classer les huiles et n'est pas systématiquement liée à l'intensité des descripteurs, celle-ci pouvant être différente d'un panéliste à un autre. L'évaluation des descripteurs permet d'avoir une indication des défauts prépondérants, par exemple un fruité trop intense ou un goût de brûlé correspondant à une désodorisation insuffisante ou mal conduite.

La moyenne des notes globales définit 3 catégories : une note $>7,4$ correspond à une huile peu fruitée, sans défaut apparent donc parfaitement désodorisée ; une note comprise entre 7 et 7,4 correspond à un fruité plus marqué acceptable, mais sans défaut rédhibitoire tandis qu'une note $<7$ correspond à une huile au goût inacceptable pour une huile désodorisée (fruité net, défaut caractérisé) ; I'huile ne peut donc pas être commercialisée.

\section{Détermination des composés volatils}

Le dosage des composés volatils est réalisé par la technique du " head space dynamique " à l'aide d'un ensemble comprenant un chromatographe en phase gazeuse et système de désorption et piégeage (Thermal Desorption Cold Trap) de Chrompack (Varian, Les Ulis, France). L'échantillon (5 mul) est déposé sur de la laine de verre silanisée et placé dans un tube de désorption en pyrex. Les 
composés volatils présents sont désorbés à $140{ }^{\circ} \mathrm{C}$ pendant $15 \mathrm{~min}$ par balayage à I'hélium (10 $\mathrm{ml} / \mathrm{min}$ ) et piégés dans un tube capillaire de silice recouvert de CP Sil 5 CB 5 mum refroidi à $140{ }^{\circ} \mathrm{C}$ par de l'azote liquide. On s'est assuré préalablement que les conditions de désorption retenues ne provoquaient pas la formation de composés volatils. Les composés volatils sont ensuite injectés à $250{ }^{\circ} \mathrm{C}$ sur une colonne capillaire $50 \mathrm{~m} \times 0,32 \mathrm{~mm}$ revêtue d'un film de 1 mum de BP1 (SGE, Courtabœuf, France), élués dans les conditions suivantes : $4{ }^{\circ} \mathrm{C} / \mathrm{min}$ de $50{ }^{\circ} \mathrm{C}$ à $200{ }^{\circ} \mathrm{C}, 10^{\circ} \mathrm{C} / \mathrm{min}$ de $200^{\circ} \mathrm{C}$ à $280^{\circ} \mathrm{C}$ puis $5 \min$ à $280^{\circ} \mathrm{C}$.

Les composés volatils dosés en $\mathrm{mg} / \mathrm{kg}$ de $\mathrm{t}$-2-hexenal par étalonnage externe ont été classés en 3 catégories par souci de simplification. La première, composée de molécules à chaîne courte et de $\mathrm{t}$-2hexenal correspond à un temps de rétention maximum de $10 \mathrm{~min}$ dans nos conditions chromatographiques. La seconde contenant principalement le t-2-heptenal, les c-t et t-t-2,4 décadiénals correspond à un temps de rétention compris entre 10 et $35 \mathrm{~min}$.

La troisième contient des molécules moins volatiles éluant avant les acides gras libres et certains composants de l'insaponifiable (temps de rétention $>35 \mathrm{~min}$ ).

\section{Détermination des tocophérols}

La teneur et la composition des tocophérols ont été déterminées par chromatographie liquide haute pression sur un ensemble de 2 cartouches de silice (Chromspher Si 5 mum) de $100 \mathrm{~mm} \times 3 \mathrm{~mm}$ en utilisant le butylhydroxyanisol (BHA) comme étalon interne et un mélange hexane/tétrahydrofurane (98:2) à $0,5 \mathrm{ml} / \mathrm{min}$ comme phase mobile. La détection est réalisée par fluorimétrie à l'aide d'un détecteur JASCO 820. Les teneurs en tocophérols (tableau 1) sont significativement plus faibles après redésodorisation du fait des conditions de vide adoptées lors de la désodorisation [3].

\section{Nez électronique}

Le nez électronique est un instrument constitué d'un ensemble de capteurs sensibles à la présence de molécules volatiles spécifiques et d'un système de traitement des signaux résultants capable de comparer à l'aide de méthodes statistiques les profils obtenus à ceux produits par des échantillons de même nature [4-8]. II s'agit donc de générer les odeurs dans des conditions réalistes et reproductibles, une partie des substances volatiles étant ensuite entraînée par un gaz inerte sur la barrette de capteurs.

Chaque capteur répondra de façon plus ou moins intense aux constituants volatils de l'espace de tête, le faisceau de données générant ainsi un profil caractéristique de l'odeur de l'échantillon. On peut alors différencier un échantillon d'un autre à l'aide de technique d'analyses de données comme l'analyse en composantes principales.

II existe un grand nombre de capteurs disponibles commercialement qu'on peut regrouper en 2 groupes.

Les capteurs chauffés (capteurs à base d'oxyde métallique) et les capteurs non chauffés (polymères conducteurs et capteurs à quartz piezoélectrique).

Les capteurs chauffés sont constitués d'un substrat en céramique sur lequel est déposé un matériau semi-conducteur constitué d'un oxyde métallique. L'élément de céramique placé entre deux 
électrodes est maintenu à haute température $\left(300-550^{\circ} \mathrm{C}\right)$ par passage d'un courant. Les substances volatiles adsorbées à la surface provoquent à la suite de réactions redox des changements de conductivité du capteur.

Les nez électroniques peuvent donc être un outil intéressant de contrôle qualité puisqu'ils sont à même de comparer rapidement le profil donné par une huile à celui donné par une autre. Ils ont d'ailleurs été exploités dans ce domaine [10,11].

II reste à s'assurer que les produits volatils adsorbés sont corrélés au goût de l'huile ou au moins à certains défauts spécifiques.

Les capteurs non chauffés n'ont pas été retenus dans notre étude car ils se sont révélés sensibles à I'humidité relative et non discriminants dans le cas des huiles raffinées contrairement à ce qu'ont observé certains auteurs [9].

Nous avons utilisé le nez électronique 5000 de Marconi Applied Technologies (Chelmsford, Essex, UK) constitué d'un module de 8 capteurs à semi-conducteurs et d'un passeur d'échantillon CTC PAL SYST (Interscience, Louvain-la-Neuve, Belgique). Pour des raisons de confidentialité, la nature de la composition des capteurs utilisés n'a pas été fournie par le constructeur.

Des essais préliminaires ont permis d'optimiser les conditions de génération de l'espace de tête. Les conditions suivantes ont été adoptées.

Deux grammes d'huile étaient pesés à $0,05 \mathrm{~g}$ près dans un flacon type pénicilline de $20 \mathrm{ml}$ fermé par un septum scellé par une bague d'aluminium. La pesée étant réalisée de façon à éviter qu'il y ait des gouttelettes d'huiles sur les parois du tube ou sur le septum.

Le temps d'incubation dans le passeur était de $15 \mathrm{~min}$, la température ayant été fixée à $80^{\circ} \mathrm{C}$ pour favoriser la désorbtion de substances volatiles. Avant injection de l'espace de tête, le flacon était agité à 500 tours par minute pendant 5 secondes.

Cinq millilitres d'espace de tête sont injectés au travers d'une boucle puis la seringue est balayée par de l'air sec (humidité $<10 \mathrm{mg} / \mathrm{kg}, \mathrm{CO}_{2}<2 \mathrm{mg} / \mathrm{kg}, \mathrm{CH}_{4}<2 \mathrm{mg} / \mathrm{kg}$ ) pendant 2 min pour éviter toute contamination ultérieure.

La lecture des réponses des capteurs peut se faire en mode dynamique ou statique. Nous avons opté pour le mode statique. Dans ce cas, les substances volatiles sont bloquées pendant 2 min dans la cellule contenant les capteurs sans qu'il y ait de balayage.

Les changements des valeurs de résistance de chaque capteur sont exprimés en pourcent de la résistance initiale.

Les valeurs enregistrées à 120 secondes sont alors traitées par le système informatique par analyse factorielle en composantes principales.

Deux mesures sur des flacons vides sont effectuées avant chaque série d'une quinzaine d'échantillons pour s'assurer de l'absence de réponse parasite. 


\section{Résultats}

\section{Détermination des volatils totaux}

La teneur en substances volatiles dosées par notre technique ne dépasse pas $120 \mathrm{mg} / \mathrm{kg}$ quel que soit le type d'huile et son état de fraîcheur (tableau 2). Des analyses effectuées à nouveau quelques semaines après dans nos conditions ont confirmé ces résultats à $\pm 20 \%$.

Les huiles redésodorisées contiennent moins de substances volatiles comme il était prévisible mais cela reste vrai même après vieillissement naturel.

On constate qu'il n'y a aucune corrélation entre la note donnée par le panel et la teneur en volatils totaux ni même avec une des trois catégories de produits telles que nous les avons définies à partir des temps de rétention (figure 1).

Bien que cette technique ait souvent été employée pour évaluer l'état oxydatif d'une huile, elle ne paraît pas être à même d'établir si une huile désodorisée est ou non neutre de goût. En effet elle n'est pas forcément représentative du stimulus global ressenti par le panéliste. Malgré sa facilité de mise en œuvre elle ne peut donc pas se substituer à un contrôle organoleptique effectué par un panel dans le cadre d'un plan de contrôle qualité industriel.

\section{Nez électronique}

Les résultats obtenus sur les échantillons préparés ont été traités par analyse en composantes principales (ACP) (figures 2 à 7).

On constate qu'il y a quatre catégories bien distinctes. Les points 1 représentent les huiles désodorisées industriellement, les points 2 les huiles redésodorisées tandis que les points 11 et 21 représentent ces deux familles d'huiles après un vieillissement naturel de 10 semaines (mesures faites en double).

Cependant, un tel traitement informatique n'est pas commode en contrôle de routine puisqu'avant chaque série d'analyse, il faudrait passer des références afin de positionner les trois régions correspondant aux huiles considérées comme " acceptables" (sur la gauche du graphe), " non acceptables " (à droite) et les huiles « limites ».

L'examen des réponses de chaque capteur a montré que parmi les 8 capteurs, certains étaient beaucoup plus sensibles que d'autres. II n'y a donc pas intérêt à traiter par ACP les réponses de 8 capteurs mais à ne retenir que 1 ou 2 capteurs choisis pour leur sensibilité.

Ainsi le tableau 3 montre les coefficients de corrélation des données de 2 de ces capteurs avec la note globale du panel.

On constate que la corrélation est différente selon les huiles, acceptables pour l'huile de tournesol mais absente pour les huiles de colza et de maïs.

L'intérêt de n'utiliser qu'un ou deux capteurs pour contrôler la neutralité de goût des huiles réside dans le fait que l'on peut définir préalablement une valeur délimitant les huiles « acceptables » des « 
non acceptables " comme on le ferait lors d'un dosage physicochimique classique. De cette façon il n'y aurait plus besoin d'un équipement informatique lourd puisqu'il s'agirait de comparer la valeur obtenue par l'un des capteurs retenus à la valeur limite prédéterminée.

Pour vérifier cette hypothèse, nous avons effectué de telles mesures sur des huiles raffinées de tournesol, colza et un mélange commercialisé d'huiles de tournesol, colza et pépins de raisin. Les échantillons provenaient de différents lieux de production, certains ayant vieillis naturellement.

Un des capteurs qui nous était paru le plus sensible et le plus sélectif a été utilisé. Les coefficients de corrélation de la réponse de ce capteur avec la note de dégustation confirme l'impossibilité de I'utiliser pour l'huile de colza (figure 8) mais montre une certaine corrélation avec l'huile de tournesol et le mélange commercial (figures 9 et 10). II n'est pas étonnant que les coefficients établis avec ces échantillons soient beaucoup plus faibles que ceux mesurés précédemment (tableau 3) du fait de I'hétérogénéité des origines et de l'historique de leur vieillissement. Cet échantillonnage s'apparente plus à un contrôle classique de produits prélevés sur des points de vente qu'à un contrôle journalier de production.

En définissant une valeur limite en dessous de laquelle l'échantillon est considéré comme " acceptable ", on constate dans cette série de mesures qu'il y a un pourcentage important de " faux acceptable » et de faux « non acceptable » (tableau 4).

Cependant il faut tenir compte que le panel a aussi une dispersion importante et qu'en particulier des échantillons classés comme « limite » peuvent être déclassés certains jours dans l'une ou l'autre catégorie.

En se limitant au contrôle journalier de production sur un site c'est-à-dire en évitant de prendre des échantillons dont l'évolution oxydative n'est pas connue ou provenant de modes de raffinage différents, le nez électronique devrait donner des résultats plus fiables.

À ce jour cependant, le nez électronique quel que soit le type de capteurs actuellement disponibles ne peut remplacer un panel pour le contrôle organoleptique des huiles végétales raffinées mais pourrait être un outil très pratique pour alléger la charge de travail du contrôle qualité en milieu industriel.

Reçu le 19/4/2002, accepté le 20/8/2002.

\section{REFERENCES}

1. BERSET C, CUVELIER ME (1996). Méthodes d'évaluation du degré d'oxydation des lipides et de mesures du pouvoir antioxydant. Science des Aliments. 16 : 219-45.

2. MAC FARLANE N, SALT J, BIRKIN R, KENDRICK A (2001). The FAST index: a poisson scale. Inform, 12 : 244-9.

3. HENON G, VIGNERON PY, STOCLIN B, CAIGNIEZ J (2001). Rapeseed oil deodorization study using the response surface methodology. Eur J Lipid Sci Technol, 103 : 467-76. 
4. BARTLETT PN, ELLIOTT JM, GARDNER JW (1997). Electronic noses and their application in the food industry. Food Technology, $51:$ 44-9.

5. MIELLE P (1996). Electronic noses: the objective instrumental characterization of food aroma. Trends in Food Science and Technology, 7 : 432-8.

6. GIESE J (2000). Electronic noses. Food Technology, 54 : 96-8.

7. STRIKE DJ, MEIJERINK MGH, KOUDELKA-HEP M (1999). Electronic noses: a mini review. Fresenius J Anal Chem, $364:$ 499-505.

8. DICKINSON TA, WHITE J, KAVER JS, WALT DR (1998). Current trends in artificial nose technology. Tibtech, $16: 250-8$.

9. SHEN N, MOIZUDDIN S, WILSON L, DUVICK S, WHITE P, POLLAK L (2001). Relationship of electronic nose analyses and sensory evalution of vegetable oils during storage. J Am Oil Chem Soc, 78 : 937-40.

10. SHIERS V, ADECHY M (1998). Use of multisensor array devices to attempt to predict shelf-lives of edible oils. Seminars Foods Analysis, $3: 43-52$.

11. BAZZO S, LOUBET F, TSUNG TAN T, HEWITT-JONES JD, ENGELEN-CORNAX CEM, QUADT JFA (1998). Quality control of edible oil using an electronic nose. Seminars Foods Analysis, 3 : 15-25.

Illustrations

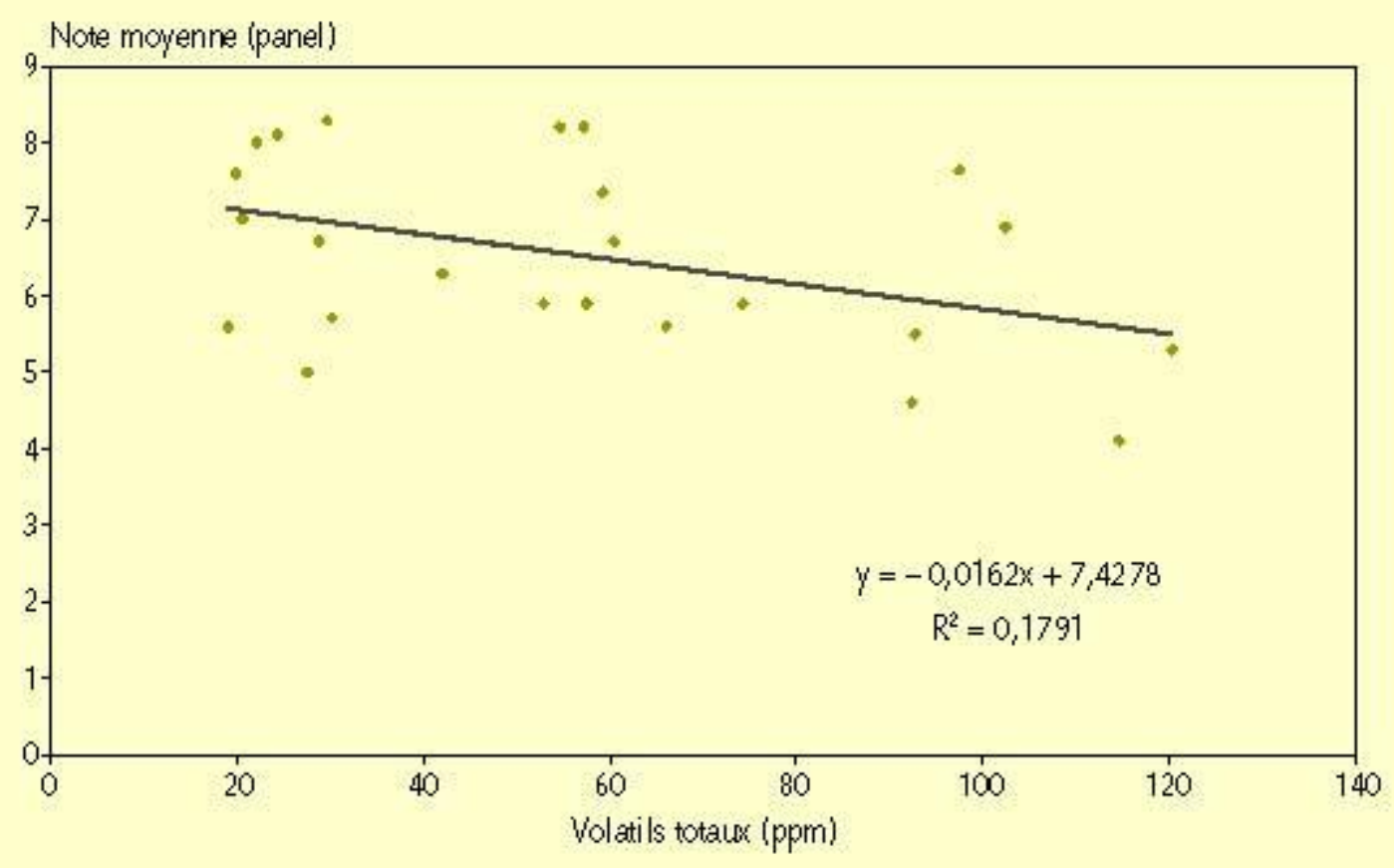

Figure 1. Corrélation note globale de dégustation teneur totale en composés volatils. 


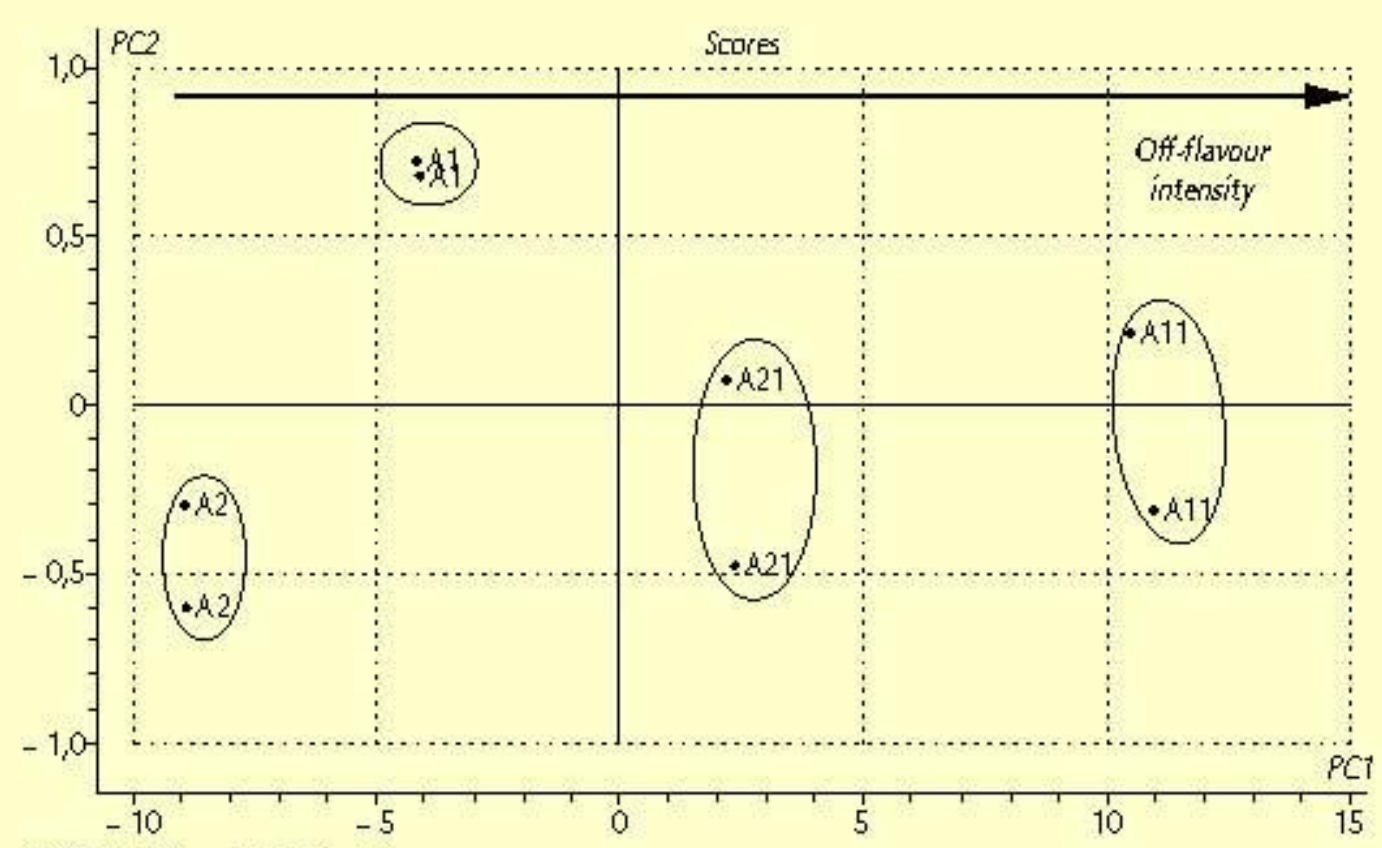

RESULT3, X-expl : $99 \%, 0 \%$

Figure 2. ACP sur huile d'arachide.

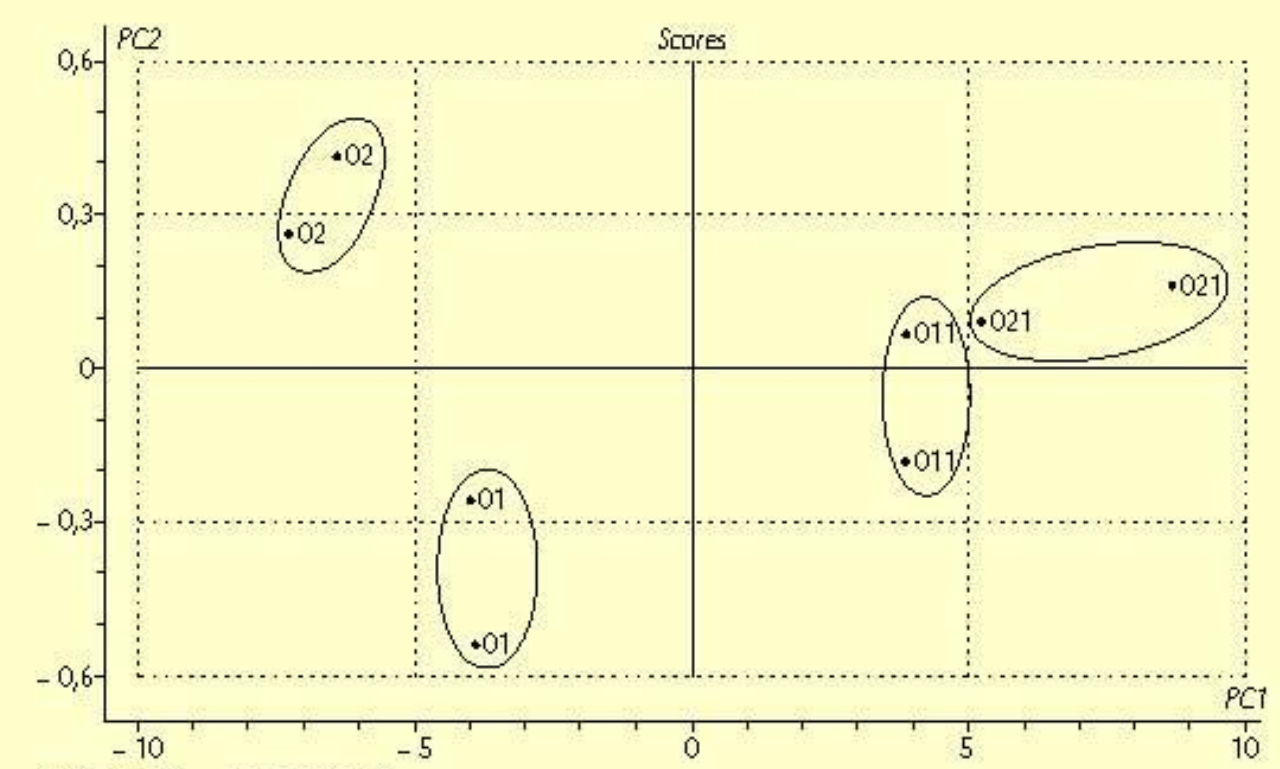

RESULT4, X-expl : $100 \%, 0 \%$

Figure 3. ACP sur huile de tournesol haut oléique. 


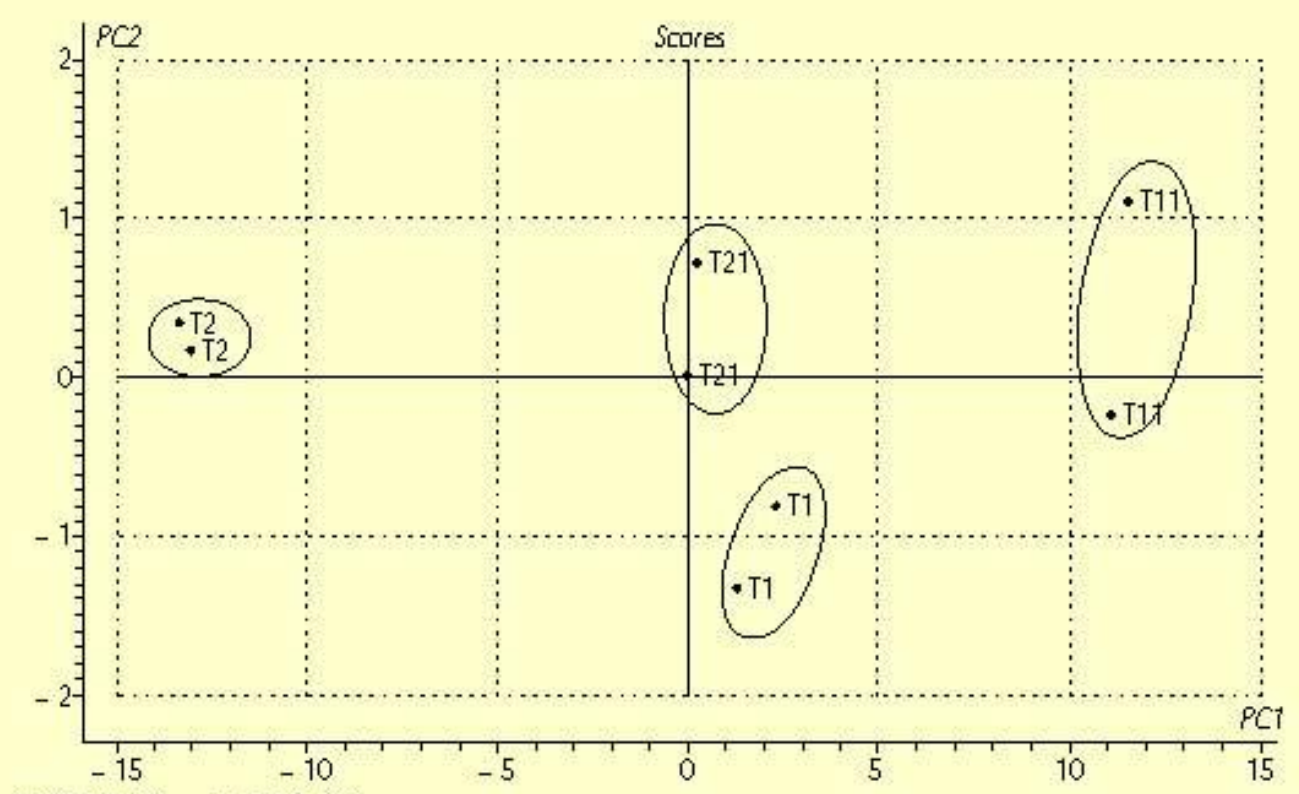

RESULT5, X-expl : $99 \%, 1 \%$

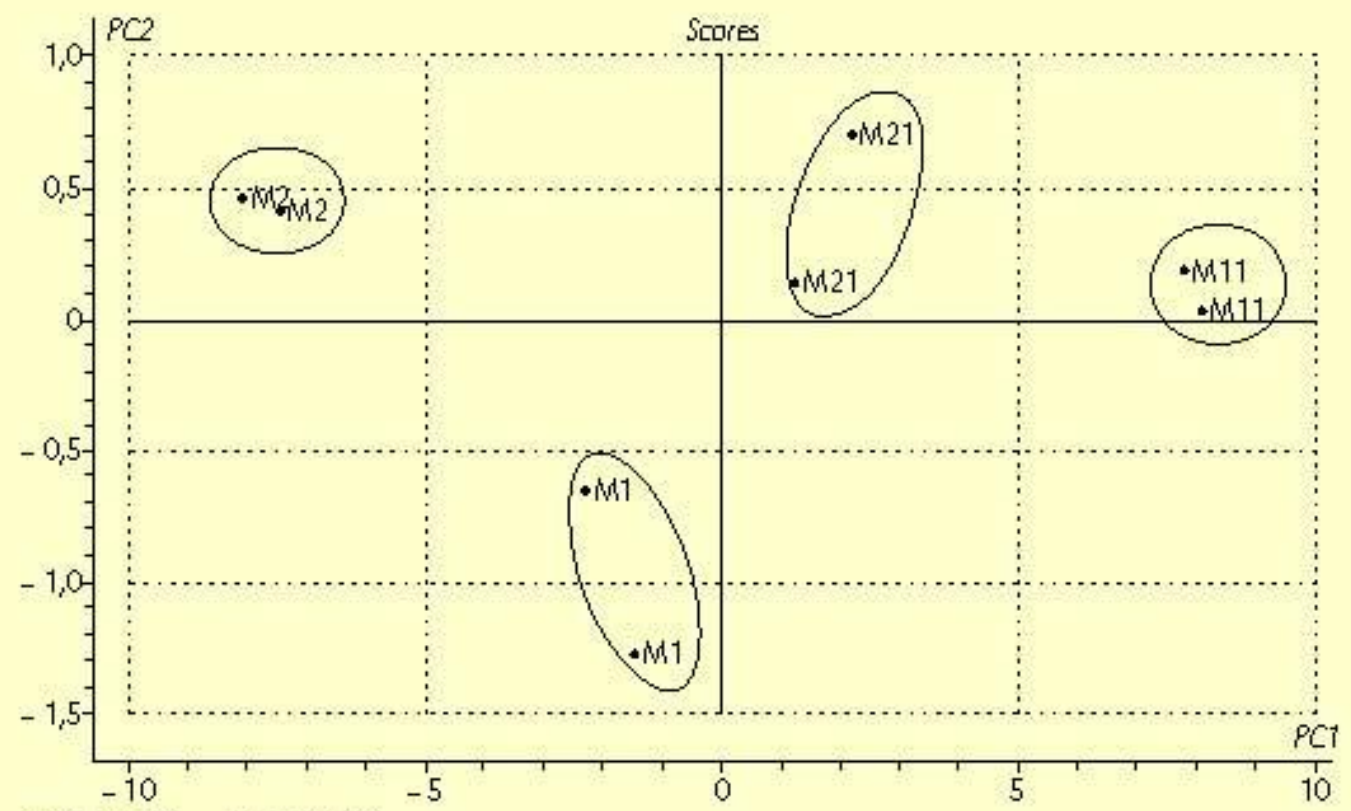

RESULT6, X-expl : $99 \%, 1 \%$

Figure 5. ACP sur huile de maïs. 


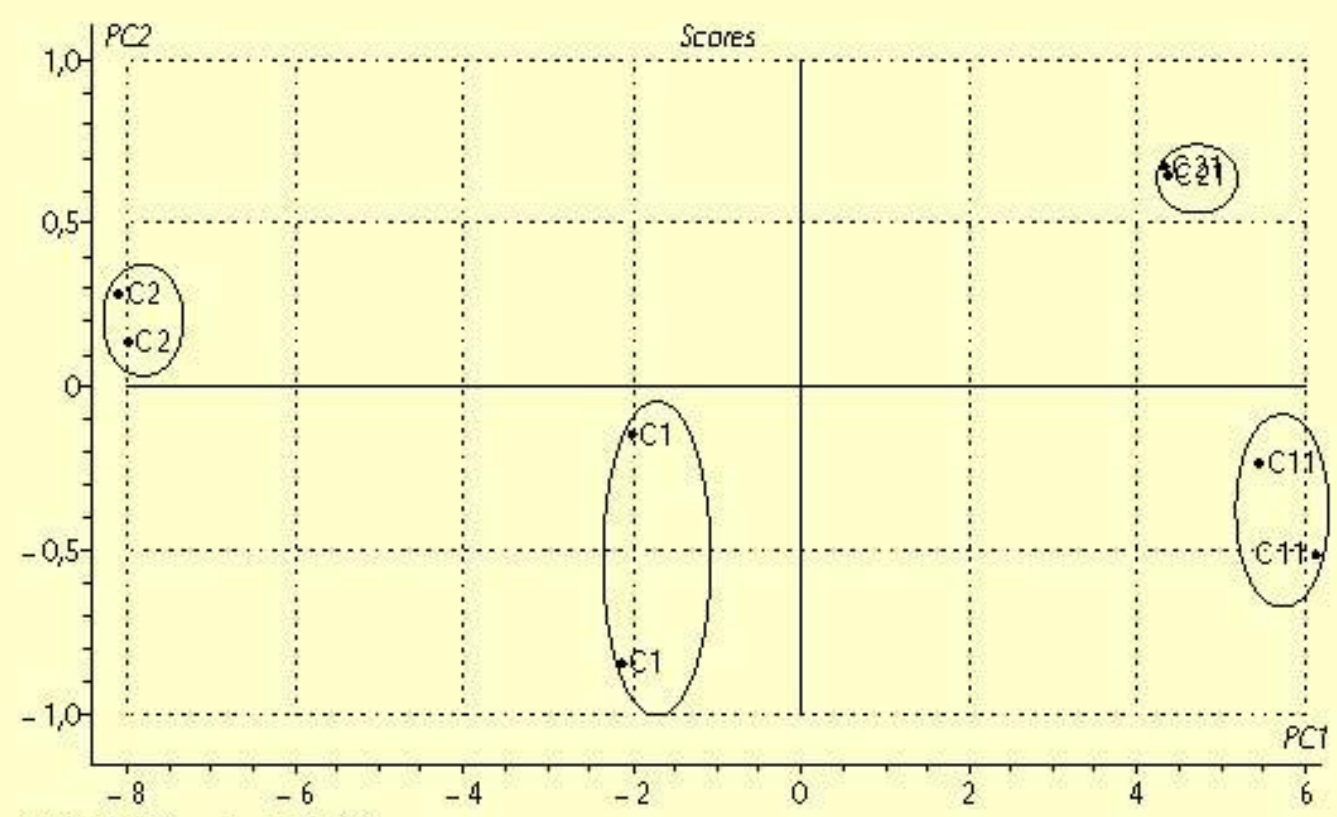

RESULT1, X-expl : $99 \%, 1 \%$

Figure 6. ACP sur huile de colza.

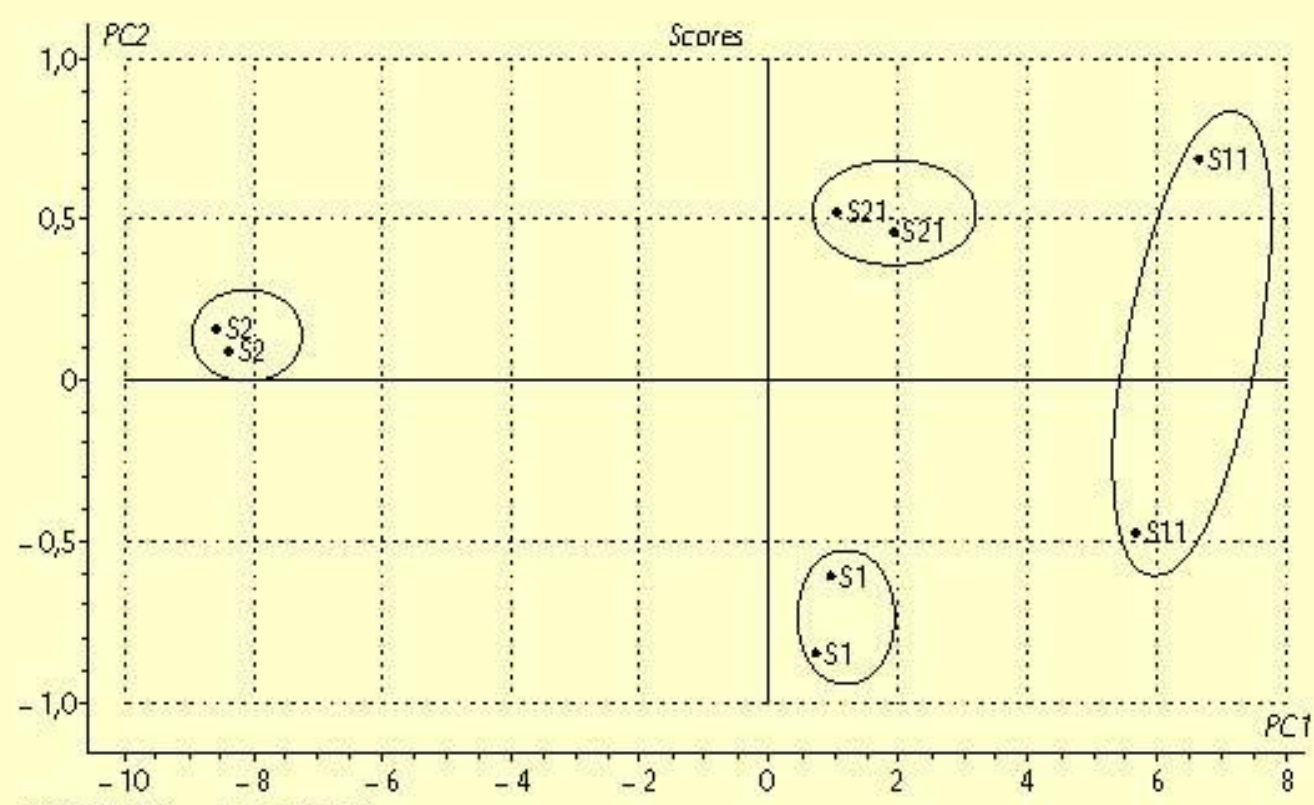

RESULT2, X-expl : $99 \%, 1 \%$

Figure 7. $A C P$ sur huile de soya. 


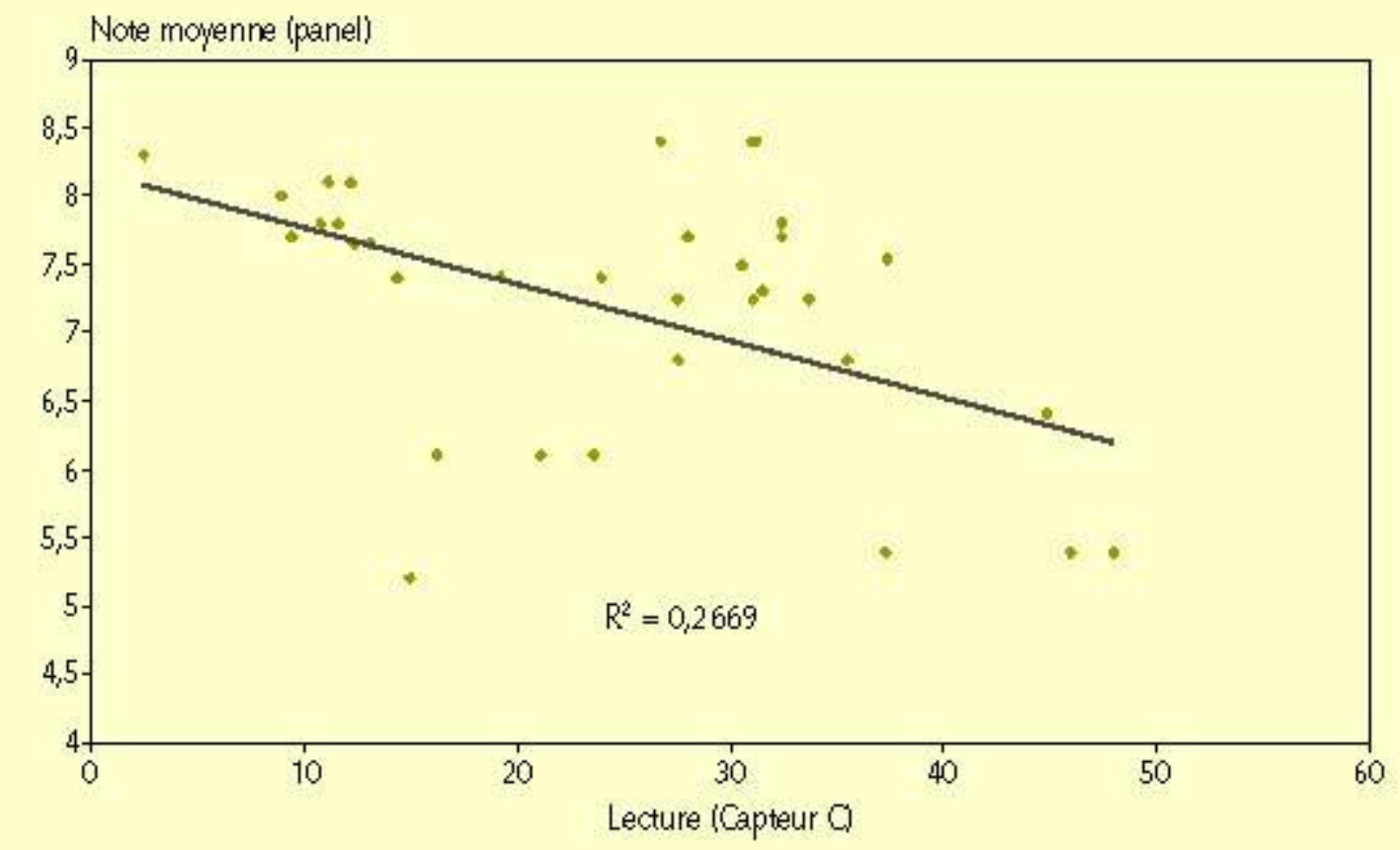

Figure 8. Huile de colza : corrélation note de dégustation vs réponse d'un capteur.

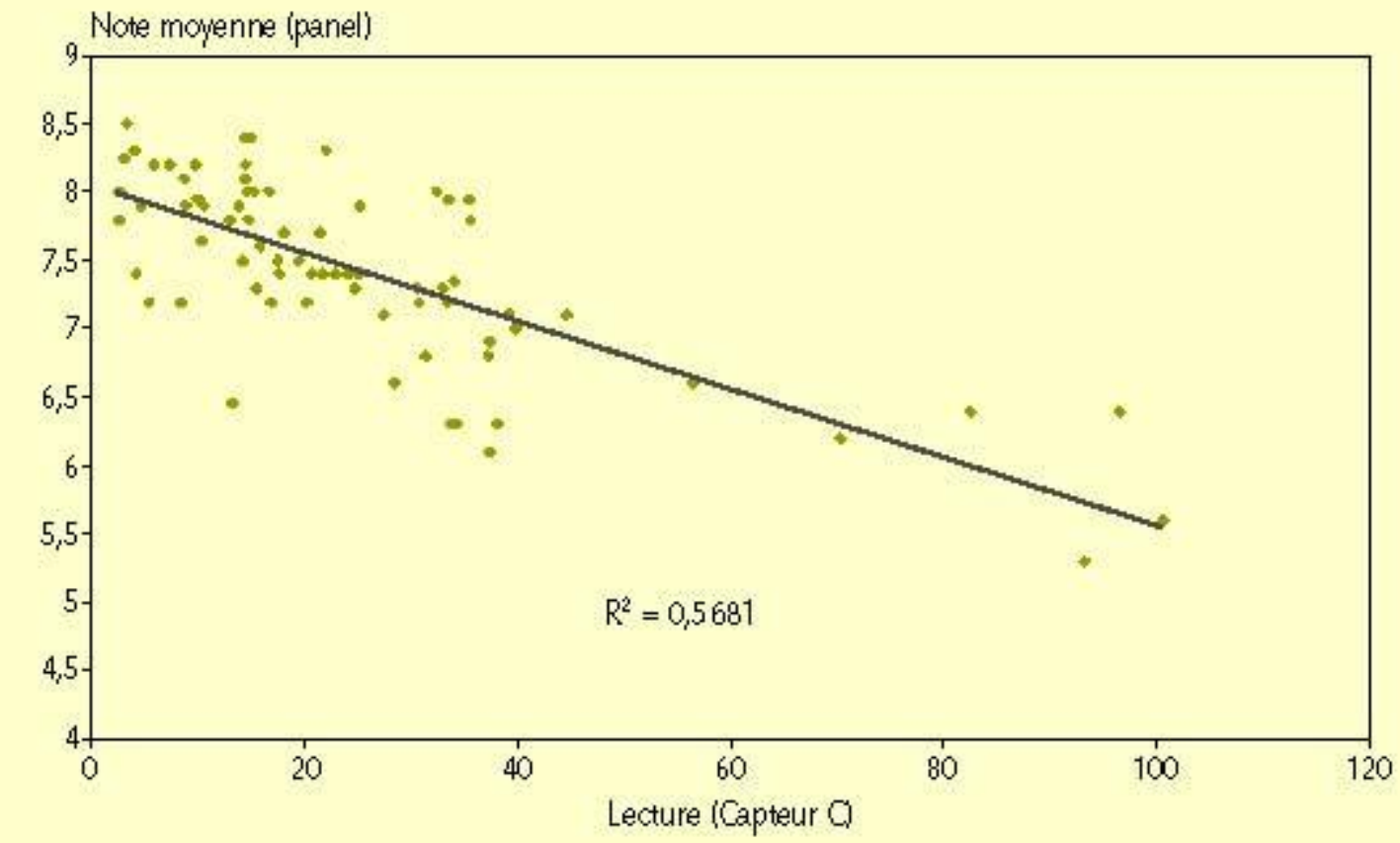

Figure 9. Huile de tournesol : corrélation note de dégustation vs réponse d'un capteur. 


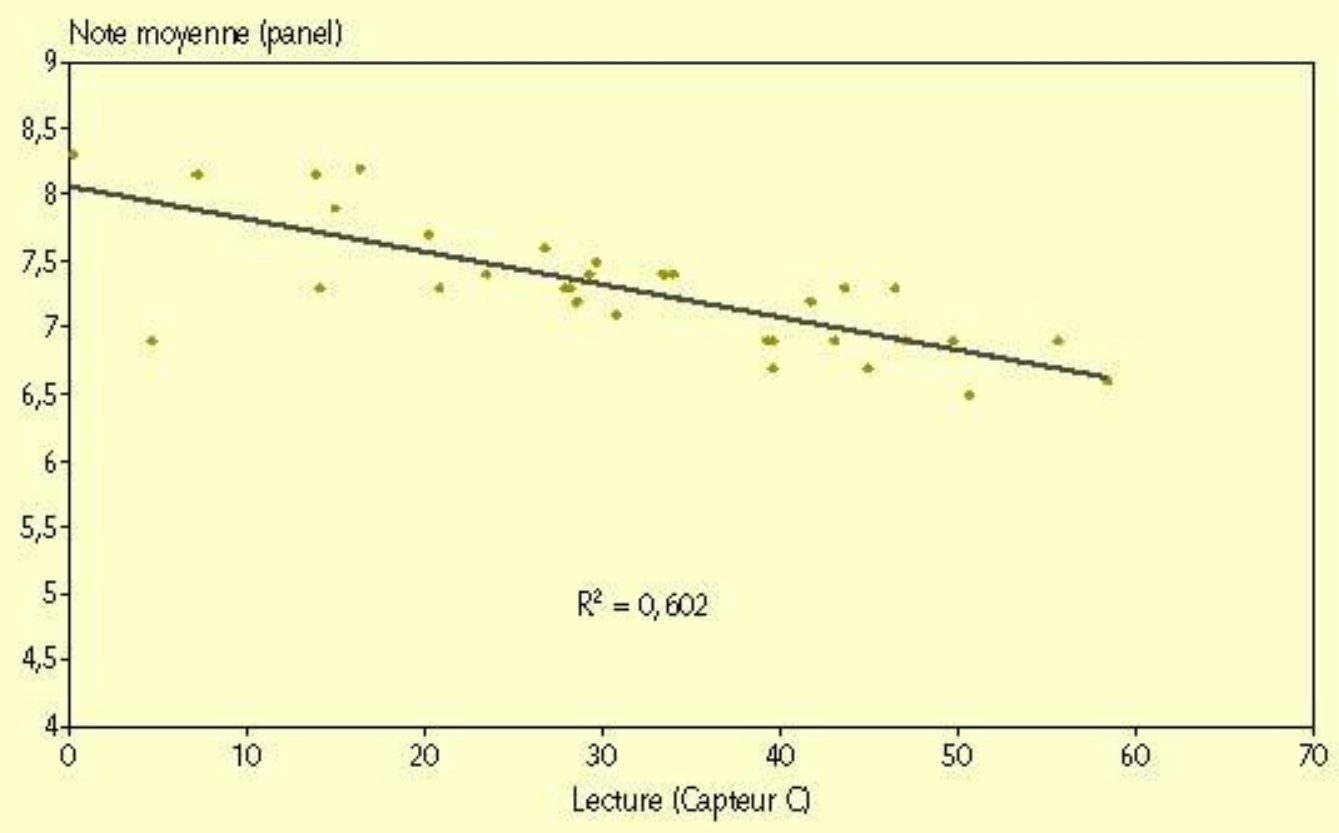

Figure 10. Mélange commercial : corrélation note de dégustation vs réponse d'un capteur.

Tableau 1. Teneurs en tocophérols des huiles désodoriséex.

\begin{tabular}{|c|c|c|}
\hline & Ed antillons & $\begin{array}{c}\text { Total } \\
(\mathrm{mg} / 100 \mathrm{~g})\end{array}$ \\
\hline \multirow{4}{*}{$\begin{array}{l}\text { Huile } \\
\text { d'arachide }\end{array}$} & A1 - raffinée & 35,8 \\
\hline & A11 - A1 après 10 semaines & 33,25 \\
\hline & A2 - redésodorisée & 19,25 \\
\hline & $A 21-A 2$ après 10 semaines & 17,3 \\
\hline \multirow{4}{*}{$\begin{array}{l}\text { Huile de } \\
\text { tournesol } \\
\text { haut oléique }\end{array}$} & Ol - raffinée & 71,2 \\
\hline & 011 - 01 après 10 semaines & 67,75 \\
\hline & 02 - redésodorisée & 47,1 \\
\hline & $021-02$ après 10 semaines & 42,65 \\
\hline \multirow{4}{*}{$\begin{array}{l}\text { Huile de } \\
\text { tournesol }\end{array}$} & T1 - raffinée & 68 \\
\hline & T11 - T1 a près 10 semaines & 71,9 \\
\hline & T2 - redésodorisée & 42,35 \\
\hline & T21 - T2 a près 10 semaines & 40,4 \\
\hline \multirow{4}{*}{ Huile de maïs } & Ml - raffinée & 104,35 \\
\hline & Ml 1 - Ml après 10 semaines & 1019 \\
\hline & M2 - redésodorisée & 60,35 \\
\hline & M21 - M2 après 10 semaines & 57,15 \\
\hline \multirow{4}{*}{ Huile de co zza } & $\mathrm{Cl}$ - raffinée & 59,5 \\
\hline & $\mathrm{C} 11$ - $\mathrm{Cl}$ après 10 sema ines & 61,5 \\
\hline & C2 - redésodorisée & 41,4 \\
\hline & $\mathrm{C} 21-\mathrm{C} 2$ après 10 sema ines & 39,7 \\
\hline \multirow{4}{*}{ Huile de soya } & S1 - raffinée & 104,5 \\
\hline & S11 - S1 après 10 emaines & 104,15 \\
\hline & S2 - redésodo risée & 60,05 \\
\hline & S21 - S2 après 10 emaines & 57,35 \\
\hline
\end{tabular}


Tableau 2. Notes de dégustation (échelle 0-10) et teneur en composés volatils des huiles déso dorisées.

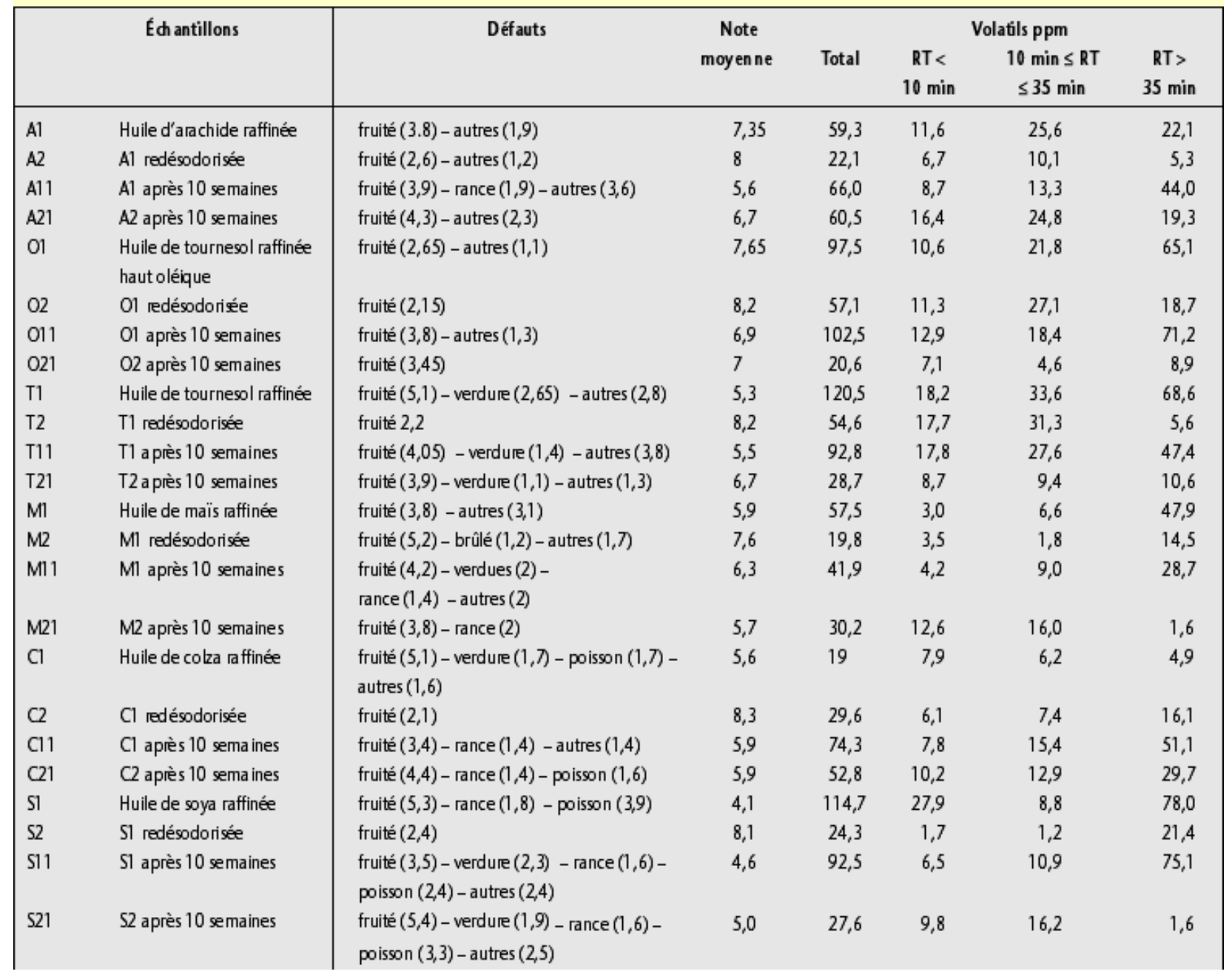

Tableau 3. Coefficient de corrélation entre la note globale du panel et la réponse de 2 capteurs.

\begin{tabular}{|l|c|c|}
\hline & $\begin{array}{c}\text { Capteur } \\
\text { A }\end{array}$ & $\begin{array}{c}\text { Capteur } \\
\text { B }\end{array}$ \\
\hline Arachide & 0,9977 & 0,9879 \\
Toumesol haut oḱique & 0,8395 & 0,8825 \\
Toumesol & 0,8188 & 0,8823 \\
Maïs & 0,3640 & 0,4988 \\
Colza & 0,6091 & 0,6794 \\
Soya & 0,8071 & 0,854 \\
Mélange commercial & 0,9393 & 0,8947 \\
\hline
\end{tabular}


Tableau 4. Pourcentage d'échantillors rejetés en appliquart une valeur limite.

\begin{tabular}{|l|c|c|c|c|c|c|}
\hline \multicolumn{5}{|c|}{ Capteur C } \\
\hline & Rejetés par E-Nose & Acceptés par le pan el & Faux « non acceptable * \% & Acceptés par E-Nose & Rejetés par le panel & Faux « acceptable * \% \\
\hline Toumesol & 12 & 58 & 20,5 & 2 & 15 & 13,5 \\
Mélange & 6 & 21 & 28 & 1 & 11 & 9 \\
commercial & & & & & &
\end{tabular}

